\title{
Distributed Decision Fusion with M-ary Signaling and k-bit Transmission on Sensor Observation in Wireless Sensor Networks
}

\author{
Victor Wen-Kai Cheng ${ }^{1, *}$, Tsang-Yi Wang ${ }^{2}$ \\ ${ }^{1}$ Department of Computer Science and Information Engineering, National Chi Nan University, Nantou, Taiwan \\ ${ }^{2}$ Graduate Institute and Communication Engineering, National Sun Yat-Sen University, Kaohsiung, Taiwan
}

\section{Email address:}

vicwk@csie.ncnu.edu.tw (Victor Wen-Kai Cheng), tcwang@mail.nsysu.edu.tw (Tsang-Yi Wang)

${ }^{*}$ Corresponding author

\section{To cite this article:}

Victor Wen-Kai Cheng, Tsang-Yi Wang. Distributed Decision Fusion with M-ary Signaling and k-bit Transmission on Sensor Observation in Wireless Sensor Networks. International Journal of Information and Communication Sciences. Vol. 6, No. 2, 2021 , pp. $30-37$. doi: 10.11648/j.ijics.20210602.12

Received: March 13, 2021; Accepted: March 30, 2021; Published: May 7, 2021

\begin{abstract}
Multi-level decisions on sensor detection is able to improve the detection performance on the final decision made at the fusion center (FC) in wireless sensor networks (WSN). In this paper, the performance analysis of an $M$-ary signaling (MS) scheme using analog transmission and a $k$-bit transmission (KB) scheme is both examined for distributed binary detection. Under the multi-level decision algorithms, each sensor sends a signal carrying the information of a quantized version of a local decision statistic such as the conditional mean or the log-likelihood ratio. In MS, the output of the quantizer is transmitted directly without digitalizing and coding process, while in $\mathrm{KB}$, each quantization output is coded with $k$ bits and hereby a sensor sends a $k$-bit hard local decision to the FC. At the FC, the linear combiner detection rule on the transmission schemes is both adopted to make the final decision. The effects of the sensor decision and the transmission errors are incorporated in the analysis of the erroneous performance of the final decision. The goal of the proposed schemes is to minimize the final errors at the FC via optimizing the region allocation on the multi-level decision at the sensor. The numerical results illustrate that the proposed schemes achieve significant improvement in error performance over the conventional schemes under either additive white Gaussian noise (AWGN) channel or Rayleigh faded channel.
\end{abstract}

Keywords: Distributed Detection, Multi-level Decision Fusion, Wireless Sensor Networks

\section{Introduction}

Distributed detection fusion using multiple sensors has been employed in a wide variety of applications in wireless sensor networks (WSN), including power spectrum sensing in cognitive radios [1-3], environmental and structural monitoring in wireless sensor networks [4-6], intruder detection in surveillance systems [7, 8], and wireless communications [9]. Among the topologies considered in the existing works, the parallel fusion network has received the most attention [10]. Conventionally, in the distributed parallel fusion network, a phenomenon in a specified environment is first observed by a sensor, and a decision based on the observation is made without exchanging the information with other local sensors. In distributed detection, the decision made by local sensors is carried out by some preliminary processing of sensor observations, usually the quantization process. Then, each sensor transmits a signal to the fusion center (FC) carrying the information of its decision in accordance with the quantization level of its observation. In the conventional system, the decision is usually binary and hence a sensor sends a quantized version of a local decision statistic, which can also be regarded as a one-bit hard local decision. The FC makes an overall decision on the phenomenon after collecting all the data from each of the sensors. The decision at the FC, however, can be erroneous due to mistakes on the detection by the sensors or the effects of channel errors.

When the decision made by each local sensor is binary, the sensor observation in the multiple-sensor system can be 
considered being recorded by each sensor via one single bit only, which is usually referred to as hard-decision fusion [10]. In contrast to the binary local decision case, many works have also studied the case of soft-decision fusion, in which the sensor observation is divided into $M$ regions, where $M$ is an arbitrary integer with $M>2[1,2,11-17]$. The work in previous study [11] considers the system in which the local sensor sends an additional quality information bit along with the binary decision bit to the FC. In the works $[1,2,12,13,14$, $18,19]$, the local soft-decision is made using log-likelihood ratio (LLR) quantizer. In the study [15], the multiple-bit local decision is made in accordance with the quantization level of received signal energy. In the works $[16,17]$, a multiple-bit decision carrying the quantized membership function of sensor observations is made by each local sensor and sent to the FC. Several other works have also studied the soft decision fusion in distributed detection system [9, 20-23].

However, the works mentioned above all assume that the information of soft local decision is digitally transmitted to the $\mathrm{FC}$, in which the information of each quantization level is mapped to several digital bits and the local softdecision (quantizer output) or the local decision performance corresponding to the received quantization level is then utilized by the FC to make its final decision, and usually Gray coding is employed in the mapping of the multiple bits. Besides, the demanding channel bandwidth in the literature [1, 2, 11-17] is scaled with the number of quantization levels. As opposed to the existing works mentioned above, this study considers both uncoded (analog) and digital transmission schemes between the local sensors and the FC. The sensor observation is divided into $M$ regions, $M \geq 2$ [26]. The information of the soft local decision in each region is sent via uncoded (analog) transmission to the FC without digitalizing and coding process, which is defined as $M$-ary signaling (MS). On the other hand, if the local decision is digitally transmitted to the FC via multiple bits, the scheme is defined as $k$-bit transmission (KB) and the number of the quantization levels on the observation is restricted to $2^{k}$. At the $\mathrm{FC}$, the linear combiner detection rule is adopted to make the final decision. Either the MS scheme or the KB scheme is aimed to achieve higher information recorded towards the phenomenon in the environment at the sensor and lower error probability under the same energy consumption on the final decision at the FC.

In the proposed MS scheme, the detection on the phenomenon is divided into $M$ equally spacing regions, where $M$ is an arbitrary integer with $M \geq 2$. When the detection falls within a specific region, a conditional mean is adopted as a representation of the observation in the region, and an uncoded transmission carrying the information of the quantization is sent to the FC [26]. The goal of the proposed scheme is to minimize the decision errors at the FC via optimizing the region allocation, i.e., varying the width of each of the $M$ regions [26]. The error performances using maximuma-posteriori (MAP) and equal-gain-combining (EGC) fusion rules at the FC are both analyzed. Nevertheless, in the proposed $\mathrm{KB}$ scheme, a $k$-bit sequence encoded from the quantization, instead of the value of the quantization itself, is sent to the FC. Both Gray code and regular binary code are employed in the encoding of the $k$ bits. At the FC, the hard decision is performed first on each of the transmitted bits and EGC is adopted on the hard-limited data to make the final decision. Similarly, the main aim is to minimize the final errors at the FC via optimizing the region allocation. Furthermore, the performance comparison of the MS and the KB schemes is conducted with the signal transmitted over either additive white Gaussian noise (AWGN) channel or Rayleigh faded channel. The proposed schemes are illustrated with numerical examples to show their significant improvement in error performance and increased information available at the FC as compared with the conventional schemes.

The remainder of this paper is organized as follows. Section 2 will first depict the MS and the KB mechanisms for binary distributed detection problem. This is followed by the performance analyses for the corresponding schemes in Section 3. Section 4 presents the results of MS and KB and their comparison, and Section 5 concludes this work.

\section{Design of Multi-level Decision Scheme}

In a WSN environment under binary hypothesis $H_{0}$ and $H_{1}$ shown in Figure 1, we assume a phenomenon $A$ either $H_{0}$ : $A=\gamma$ or $H_{1}: A=-\gamma$, where $\gamma>0$, occurs symmetrically with equal prior probability $P\left[H_{0}\right]=P\left[H_{1}\right]=0.5$.

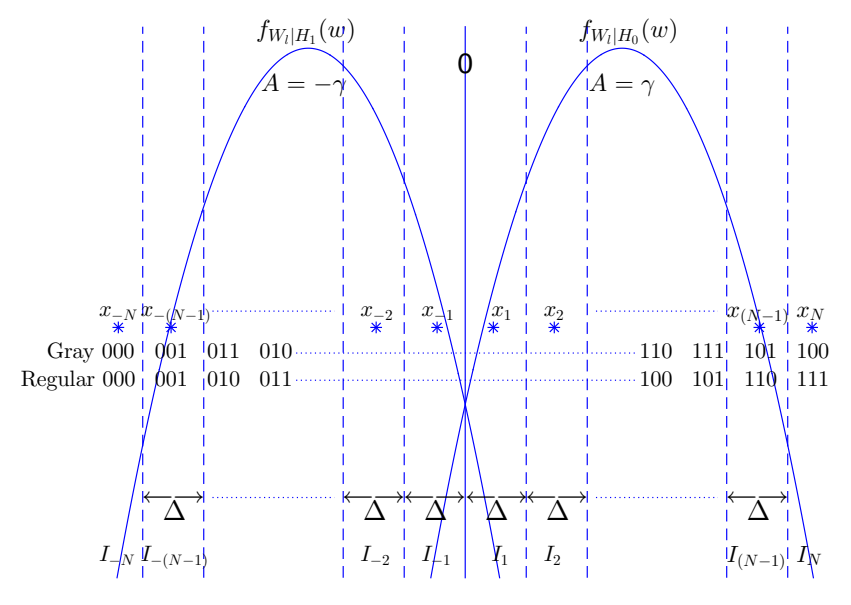

Figure 1. WSN with multi-level decision scheme on sensor detection

$L$ sensors observe the measurements generated from $H_{0}$ or $H_{1}$ and the observation value by the $l$-th sensor is denoted by $W_{l}, l=1, \ldots, L$, independent and identically distributed (i.i.d.) from sensor to sensor. Also, assume $H_{0}: W_{l}=\gamma+$ $\eta_{l} ; H_{1}: W_{l}=-\gamma+\eta_{l}$, where $\eta_{l}$ is a standard normal random variable. The probability density function (PDF) of $W_{l}$ conditioned on $H_{0}$ or $H_{1}$ is $f_{W_{l} \mid H_{0}}(w)=e^{-(w-\gamma)^{2} / 2} / \sqrt{2 \pi}$ or $f_{W_{l} \mid H_{1}}(w)=e^{-(w+\gamma)^{2} / 2} / \sqrt{2 \pi}$. In the considered parallel fusion networks, the $l$-th sensor makes its own binary decision $X$, independent of all other nodes. Conventionally, the binary decision is $X= \pm 1$ and the signal $s_{l}$ transmitted to the FC by the $l$-th sensor is $s_{l}=\left\{\begin{array}{cc}\sqrt{E}, & W_{l}>0 \\ -\sqrt{E}, & W_{l}<0\end{array}\right.$, where 
$E$ is the energy of the transmitted signal. In addition, an identical local decision rule is assumed among all the sensors. An erroneous decision at the sensor occurs with probability $P\left[W_{l}<0 \mid H_{0}\right]=P\left[W_{l}>0 \mid H_{1}\right]=Q(\gamma)$, where $Q(\gamma)$ is the standard normal complementary cumulative distribution function. The received signal from the $l$-th sensor at the FC is $R_{l}=s_{l}+n_{l}$, where $n_{l}$ is the Gaussian noise in the AWGN channel. All $n_{l}$ 's are assumed i.i.d. with $\mathrm{E}\left[n_{l}\right]=0$ and $\operatorname{Var}\left[n_{l}\right]=N_{0} / 2$ for $l=1,2, \ldots, L$. The final decision made at the $\mathrm{FC}$ is

$$
\begin{aligned}
\sum_{l=1}^{L} R_{l}>0 & \Rightarrow \text { Decide } H_{0} \\
\sum_{l=1}^{L} R_{l}<0 & \Rightarrow \text { Decide } H_{1}
\end{aligned}
$$

and its error probability $P_{e}$ is $[24,25]$

$$
\begin{aligned}
P_{e} & =P\left[\sum_{l=1}^{L} R_{l}<0 \mid H_{0}\right]=P\left[\sum_{l=1}^{L} R_{l}>0 \mid H_{1}\right] \\
& =\sum_{i=0}^{L}\left({ }_{i}^{L}\right) Q(\gamma)^{i}(1-Q(\gamma))^{L-i} Q\left((L-2 i) \sqrt{\frac{2 E}{L N_{0}}}\right) .
\end{aligned}
$$

Moreover, if the transmitted signal to the FC by the $l$-th sensor is considered one single bit $u_{l}, u_{l}=$ $\left\{\begin{array}{ll}1, & s_{l}=\sqrt{E} \\ 0, & s_{l}=-\sqrt{E}\end{array}\right.$, the hard decision rule is first applied on the received signal $R_{l}$, and the decoded bit $\hat{u}_{l}$ is $\hat{u}_{l}=$ $\left\{\begin{array}{ll}1, & R_{l}>0 \\ 0, & R_{l}<0\end{array}\right.$. The bit error probability is $\mathrm{P}\left[\hat{u}_{l} \neq u_{l}\right]=$ $Q\left(\sqrt{\frac{2 E}{N_{0}}}\right)$. The final decision made at the FC is via the majority rule.

$$
\begin{aligned}
& \sum_{l=1}^{L} \hat{u}_{l}>\frac{L}{2} \Rightarrow \text { Decide } H_{0} \\
& \sum_{l=1}^{L} \hat{u}_{l}=\frac{L}{2} \Rightarrow \text { Randomly decide } H_{0} \text { or } H_{1} \\
& \sum_{l=1}^{L} \hat{u}_{l}<\frac{L}{2} \Rightarrow \text { Decide } H_{1} .
\end{aligned}
$$

The error probability $P_{e}$ is [24, 25]

$$
P_{e}=\left\{\begin{array}{r}
\sum_{i=\frac{L+1}{2}}^{L}\left(\begin{array}{l}
L \\
i
\end{array}\right) Q\left(\sqrt{\frac{2 E}{N_{0}}}\right)^{i}\left(1-Q\left(\sqrt{\frac{2 E}{N_{0}}}\right)\right)^{L-i} \\
\sum_{i=\frac{L+1}{2}}^{L}\left(\begin{array}{l}
L \\
i
\end{array}\right) Q\left(\sqrt{\frac{2 E}{N_{0}}}\right)^{i}\left(1-Q\left(\sqrt{\frac{2 E}{N_{0}}}\right)\right)^{L-i} \\
+0.5\left(\begin{array}{l}
L \\
\frac{L}{2}
\end{array}\right) Q\left(\sqrt{\frac{2 E}{N_{0}}}\right)^{\frac{L}{2}}\left(1-Q\left(\sqrt{\frac{2 E}{N_{0}}}\right)\right)^{\frac{L}{2}} \\
(L \text { even })
\end{array}\right.
$$

\section{1. $M$-ary Signaling}

A multi-level decision algorithm is applied on the observation at each sensor $[24,25,26]$, where $W_{l}$ at the $l$-th sensor is divided into $M$ equally spacing regions as shown in Figure 1. When $M=2 N$ is even, the $2 N$ regions are $I_{-N}, I_{-(N-1)}, \ldots, I_{-1}, I_{1}, \ldots, I_{N}$, and the $(2 N-1)$ boundaries are $-(N-1) \Delta,-(N-$ 2) $\Delta, \ldots,-\Delta, 0, \Delta, \ldots,(N-1) \Delta . \quad \Delta$ is the width of each region except for the two end regions $I_{-N}$ and $I_{N}$. Similarly, when $M=(2 N+1)$ is odd, the $(2 N+$ 1) regions are $I_{-N}, I_{-(N-1)}, \ldots, I_{-1}, I_{0}, I_{1}, \ldots, I_{N}$, and the $2 N$ boundaries are $-\left(N-\frac{1}{2}\right) \Delta,-\left(N-\frac{3}{2}\right) \Delta, \ldots$, $-\frac{1}{2} \Delta, \frac{1}{2} \Delta, \ldots,\left(N-\frac{1}{2}\right) \Delta$. Define $p_{n}=P\left[W_{l} \in I_{n} \mid H_{0}\right]=$ $Q\left(d_{\text {low }}-\gamma\right)-Q\left(d_{\text {up }}-\gamma\right), n=-N,-(N-1), \ldots, N$, where $d_{\text {low }}$ and $d_{\text {up }}$ denote the lower and upper bounds of $I_{n}$, i.e., $I_{n}=\left\{W_{l} \mid d_{\text {low }}<W_{l}<d_{\text {up }}\right\}$. $\left(d_{\text {up }}-d_{\text {low }}\right)$ is $\Delta$ except for $I_{-N}$ and $I_{N}$, where $d_{\text {low }}=-\infty$ for $I_{-N}$ and $d_{u p}=\infty$ for $I_{N}$. From symmetry, $P\left[W_{l} \in I_{n} \mid H_{1}\right]=P\left[W_{l} \in I_{-n} \mid H_{0}\right]=p_{-n}$.

$M$-level quantization $X$ is applied on the observation, and $x_{n}$ is the representation value of $X$ in the $n$-th region $I_{n}$. At the $l$-th sensor, $x_{n}$ is defined as the conditional mean when $W_{l}$ falls in $I_{n}$, i.e., $x_{n}=E\left[W_{l} \mid W_{l} \in I_{n}\right]=$ $P\left[H_{0}\right] E\left[W_{l} \mid H_{0}, W_{l} \in I_{n}\right]+P\left[H_{1}\right] E\left[W_{l} \mid H_{1}, W_{l} \in I_{n}\right][26]$.

$$
\begin{aligned}
x_{n} & =\frac{1}{p_{n}+p_{-n}}\left(\frac{e^{-\left(d_{\text {low }}-\gamma\right)^{2} / 2}-e^{-\left(d_{\text {up }}-\gamma\right)^{2} / 2}}{\sqrt{2 \pi}}+p_{n} \gamma\right. \\
& \left.+\frac{e^{-\left(d_{\text {low }}+\gamma\right)^{2} / 2}-e^{-\left(d_{\text {up }}+\gamma\right)^{2} / 2}}{\sqrt{2 \pi}}-p_{-n} \gamma\right) .
\end{aligned}
$$

From symmetry, $x_{-n}=-x_{n}$ and $x_{0}=0$. Note that $I_{0}, p_{0}$ and $x_{0}$ exist only when $M$ is odd. $\left|x_{i}\right|$ increases as $\left|W_{l}\right|$ increases and vice versa. The conventional scheme is regarded as $M=2$. The signal $s_{l}$ transmitted to the FC by the $l$-th sensor is $x_{n} \sqrt{E}$, uncoded and in accordance with the decision on $W_{l} \in I_{n}$. The signal set is thus extended from $\{ \pm 1\}$, binary in the conventional fusion system, to $\left\{x_{-N}, x_{-(N-1)}, \ldots, x_{N}\right\}$, and thus $M$-ary signaling (MS) is achieved in the proposed algorithm. Given $\gamma$ and $E / N_{0}$, the goal of the proposed algorithm is to minimize the error rate $P_{e}$ of the final decision at the FC via varying $\Delta$ to obtain the optimal region allocation. The error performance is improved through the optimization, and this procedure does not exist in the conventional fusion scheme. The average consumed energy $\bar{E}$ of the transmitted signal is $\bar{E}=\sum_{n=-N}^{N} p_{n} x_{n}^{2} E$, while the consumed energy in the conventional fusion system is always $\bar{E}=E$. The error performance will be examined under the same energy spent on the transmission to the FC.

\section{2. $k$-bit Transmission}

A different approach on the transmission by the $l$-th sensor is when $M=2^{k}, k=1,2,3, \ldots$, a $k$-bit sequence based on the detection of $W_{l} \in I_{n}$., instead of the value of $x_{n}$, is transmitted to the FC. The proposed algorithm is defined as the $k$-bit transmission (KB) scheme. The transmitted signal is hence $( \pm \sqrt{E}, \pm \sqrt{E}, \ldots, \pm \sqrt{E})_{1 \times k}$, e.g., for $k=3,(000)$ is $(-\sqrt{E},-\sqrt{E},-\sqrt{E})$ and $(001)$ is $(-\sqrt{E},-\sqrt{E}, \sqrt{E})$, etc. The consumed energy $\bar{E}$ is thus $k E$. Gray code and regular binary code are both considered in the encoding of the $k$ bits, as is depicted in Figure 1 with $k=3$. Each transmitted bit is 
first hard-limit decoded at the FC and the estimation $\hat{x}_{l}$ from the $l$-th sensor is obtained for the FC to make the final decision. Similarly, $P_{e}$ is minimized via varying $\Delta$ to obtain the optimal region allocation in the $\mathrm{KB}$ scheme.

\section{Performance Analyses}

\section{1. $M$-ary Signaling}

The received signal from the $l$-th sensor at the $\mathrm{FC}$ is $R_{l}=$ $s_{l}+n_{l}$, and the PDF of $R_{l}$ is

$$
\begin{aligned}
f_{R_{l} \mid H_{0}}\left(r_{l}\right) & =\sum_{n=-N}^{N} \frac{p_{n} e^{-\left(r_{l}-x_{n} \sqrt{E}\right)^{2} / N_{0}}}{\sqrt{\pi N_{0}}} \\
f_{R_{l} \mid H_{1}}\left(r_{l}\right) & =\sum_{n=-N}^{N} \frac{p_{-n} e^{-\left(r_{l}-x_{n} \sqrt{E}\right)^{2} / N_{0}}}{\sqrt{\pi N_{0}}} \\
& =\sum_{n=-N}^{N} \frac{p_{n} e^{-\left(r_{l}+x_{n} \sqrt{E}\right)^{2} / N_{0}}}{\sqrt{\pi N_{0}}} \\
& =f_{R_{l} \mid H_{0}}\left(-r_{l}\right)
\end{aligned}
$$

The optimal MAP rule on the log-likelihood $[25,26]$ is applied to obtain the final decision $\hat{A}= \pm \gamma$, i.e., $H_{0}$ or $H_{1}$.

$$
\sum_{l=1}^{L} \log \left(f_{R_{l} \mid H_{0}}\left(r_{l}\right)\right) \underset{\substack{\hat{A}=\gamma \\ \hat{A}=-\gamma}}{\gtrless} \sum_{l=1}^{L} \log \left(f_{R_{l} \mid H_{1}}\left(r_{l}\right)\right) .
$$

Without loss of generality, assume $H_{0}$ occurs, i.e., $A=\gamma$, and thus the error probability $P_{e}$ of the final decision is

$$
P_{e}=P\left[\sum_{l=1}^{L} \log \left(\frac{f_{R_{l} \mid H_{0}}\left(r_{l}\right)}{f_{R_{l} \mid H_{1}}\left(r_{l}\right)}\right)\right]<0
$$

Given $\gamma, N, L, \Delta$, and $E / N_{0}, P_{e}$ is derived via numerical analysis, and is optimized, i.e., minimized, by revising $\Delta$ through changing the boundaries of $I_{n}$ 's iteratively.

The knowledge of $p_{n}$ is, however, a requirement for the MAP rule. When the information of $p_{n}$ is unavailable at the FC, a sub-optimal EGC rule $[25,26]$ is adopted.

$$
\sum_{l=1}^{L} R_{l} \underset{\substack{\hat{A}=\gamma \\ \hat{A}=-\gamma}}{\gtrless} 0,
$$

and hence

$$
\begin{aligned}
P_{e} & =P\left[\sum_{l=1}^{L} R_{l}<0 \mid H_{0}\right] \\
& =\int_{-\infty}^{0} f_{R_{1} \mid H_{0}}(r) * f_{R_{2} \mid H_{0}}(r) * \ldots * f_{R_{L} \mid H_{0}}(r) d r,
\end{aligned}
$$

where the asterisk $*$ denotes the convolution of the functions. Similarly, $P_{e}$ under the EGC rule is found via numerical analysis and its optimization lies in $\Delta$.

Furthermore, when the channel between the sensor and the
FC is faded, the received signal from the $l$-th sensor becomes $R_{l}=\Lambda_{l} s_{l}+n_{l}$, where $\Lambda_{l}$ is the propagation gain and is modeled a Rayleigh random variable with the PDF $f_{\Lambda_{l}}(z)=$ $2 z e^{-z^{2}}$ and $\mathrm{E}\left[\Lambda_{l}^{2}\right]=1$, and thus $\bar{E}=\sum_{n=-N}^{N} p_{n} x_{n}^{2} E$ remains the same. Assume the fading is i.i.d. among the sensors, and the PDF of $R_{l}$ given $H_{0}$ is

$$
\begin{gathered}
f_{R_{l} \mid H_{0}}\left(r_{l}\right)=\sum_{n=-N}^{N} p_{n}\left(\frac{e^{-r_{l}^{2} / N_{0}}}{\sqrt{\pi N_{0}}\left(1+x_{n} E / N_{0}\right)}+\right. \\
\left.\frac{2 x_{n} \sqrt{E} r_{l} Q\left(-r_{l} \sqrt{\frac{2 x_{n}^{2} E}{N_{0}\left(N_{0}+x_{n}^{2} E\right)}}\right) e^{-r_{l}^{2} /\left(N_{0}+x_{n}^{2} E\right)}}{\left(N_{0}+x_{n}^{2} E\right)^{3 / 2}}\right) .
\end{gathered}
$$

When the EGC rule is applied at the FC,

$$
\begin{gathered}
P_{e}=P\left[\sum_{l=1}^{L} R_{l}<0 \mid H_{0}\right] \\
=\int_{-\infty}^{0} f_{R_{1} \mid H_{0}}(r) * f_{R_{2} \mid H_{0}}(r) * \ldots * f_{R_{L} \mid H_{0}}(r) d r
\end{gathered}
$$

and, similarly, $P_{e}$ is obtained and optimized via numerical analysis.

\section{2. $k$-bit Transmission}

When a $k$-bit sequence is transmitted by the $l$-th sensor, the received signal $R_{l}$ at the FC is $R_{l}=\left( \pm \sqrt{E}+n_{1}, \pm \sqrt{E}+\right.$ $\left.\left.n_{2}, \ldots, \pm \sqrt{E}\right)+n_{k}\right), n_{i} \sim N\left(0, N_{0} / 2\right)$ is the Gaussian noise in the AWGN channel, $i=1,2, \ldots, k$. The hard-decision rule is applied on each of the $k$ bits and the bit error probability is $Q\left(\sqrt{\frac{2 E}{N_{0}}}\right)$. The estimation $\hat{x}_{n}$ from the $k$ received bits is first made. For example, when $k=3, \hat{x}_{l}=x_{-4}$ if the decoded bits are (000), and $\hat{x}_{l}=x_{-3}$ if the decoded bits are (001), etc. The probability on $\hat{x}_{l}$ can thus be derived. For example, when $k=$ 3 and Gray code is employed,

$$
\begin{aligned}
& P\left[\hat{x}_{l}=x_{-4}\right]=p_{-4} P_{000 \rightarrow 000}+p_{-3} P_{001 \rightarrow 000} \\
& +p_{-2} P_{011 \rightarrow 000}+p_{-1} P_{010 \rightarrow 000}+p_{1} P_{110 \rightarrow 000} \\
& +p_{2} P_{111 \rightarrow 000}+p_{3} P_{101 \rightarrow 000}+p_{4} P_{100 \rightarrow 000},
\end{aligned}
$$

where $P_{000 \rightarrow 000}=\left(1-Q\left(\sqrt{\frac{2 E}{N_{0}}}\right)\right)^{3}, P_{001 \rightarrow 000}=P_{010 \rightarrow 000}=$ $P_{100 \rightarrow 000}=Q\left(\sqrt{\frac{2 E}{N_{0}}}\right)\left(1-Q\left(\sqrt{\frac{2 E}{N_{0}}}\right)\right)^{2}, P_{011 \rightarrow 000}=$ $P_{110 \rightarrow 000}=P_{101 \rightarrow 000}=Q\left(\sqrt{\frac{2 E}{N_{0}}}\right)^{2}\left(1-Q\left(\sqrt{\frac{2 E}{N_{0}}}\right)\right)$, $P_{111 \rightarrow 000}=Q\left(\sqrt{\frac{2 E}{N_{0}}}\right)^{3}$.

The majority rule is applied on the final decision at the FC.

$$
\begin{aligned}
& \sum_{l=1}^{L} \hat{x}_{l}>0 \Rightarrow \text { Decide } H_{0} \\
& \sum_{l=1}^{L} \hat{x}_{l}=0 \Rightarrow \text { Randomly decide } H_{0} \text { or } H_{1}
\end{aligned}
$$




$$
\sum_{l=1}^{L} \hat{x}_{l}<0 \Rightarrow \text { Decide } H_{1}
$$

Moreover, when the information of $p_{n}$ is shared at the FC, the estimation $\hat{x}_{l}$ from the $k$ decoded bits can be otherwise interpreted as the $\log$-likelihood in $I_{n}$. For example, when $k=$ $3, \hat{x}_{l}=\log \frac{p_{-4}}{p_{4}}$ if the decoded bits are (000), and $\hat{x}_{l}=\log \frac{p_{-3}}{p_{3}}$ if the decoded bits are (001), etc. The majority rule on the final decision remains the same under the log-likelihood estimation. The error probability $P_{e}$ of the final decision from the above is obtained via numerical analysis, and, similarly, minimized via varying $\Delta$.

When the $k$-bit sequence is transmitted over a Rayleigh faded channel, the entire $k$ bits are assumed through the same fading. When $k=1$, the bit error probability is $(0.5-$ $\left.0.5 / \sqrt{1+N_{0} / E}\right)$. When $k \geq 2$ and assume $q$ out of the $k$ bits are in error, the error probability is

$$
\int_{0}^{\infty} 2 a e^{-a^{2}} Q\left(a \sqrt{\frac{2 E}{N_{0}}}\right)^{q}\left(1-Q\left(a \sqrt{\frac{2 E}{N_{0}}}\right)\right)^{k-q} d a .
$$

For example, $k=3, q=0$,

$$
P_{000 \rightarrow 000}=\int_{0}^{\infty} 2 a e^{-a^{2}}\left(1-Q\left(a \sqrt{\frac{2 E}{N_{0}}}\right)\right)^{3} d a
$$

$k=3, q=1$,

$$
P_{000 \rightarrow 001}=\int_{0}^{\infty} 2 a e^{-a^{2}} Q\left(a \sqrt{\frac{2 E}{N_{0}}}\right)\left(1-Q\left(a \sqrt{\frac{2 E}{N_{0}}}\right)\right)^{2} d a
$$

$k=3, q=2$,

$$
P_{000 \rightarrow 011}=\int_{0}^{\infty} 2 a e^{-a^{2}} Q\left(a \sqrt{\frac{2 E}{N_{0}}}\right)^{2}\left(1-Q\left(a \sqrt{\frac{2 E}{N_{0}}}\right)\right) d a
$$

$k=3, q=3$,

$$
P_{000 \rightarrow 111}=\int_{0}^{\infty} 2 a e^{-a^{2}} Q\left(a \sqrt{\frac{2 E}{N_{0}}}\right)^{3} d a, \text { etc. }
$$

Hence, the probability on $\hat{x}_{l}$ can be derived accordingly. Similarly, the error probability $P_{e}$ of the final decision is minimized via varying $\Delta$ when the channel fading exists.

\section{Results}

$P_{e}$ of the WSN under MS with $\gamma=1$ is shown in Figure 2. $P_{e}$ is lower than that of the conventional system and is improved as $M$ increases. The error performance under the MAP rule is better than that of the EGC rule. No remarkable difference exists between the performance of the MAP rule and the EGC rule when SNR is high or when $M$ is large, and the performance remains virtually the same for $M \geq 50$. The flooring of the curves at high SNR is due to the fact that the erroneous behavior of the transmitted signal to the $\mathrm{FC}$ vanishes as SNR increases and hence the behavior is dominated by the decision errors made by the sensors only.

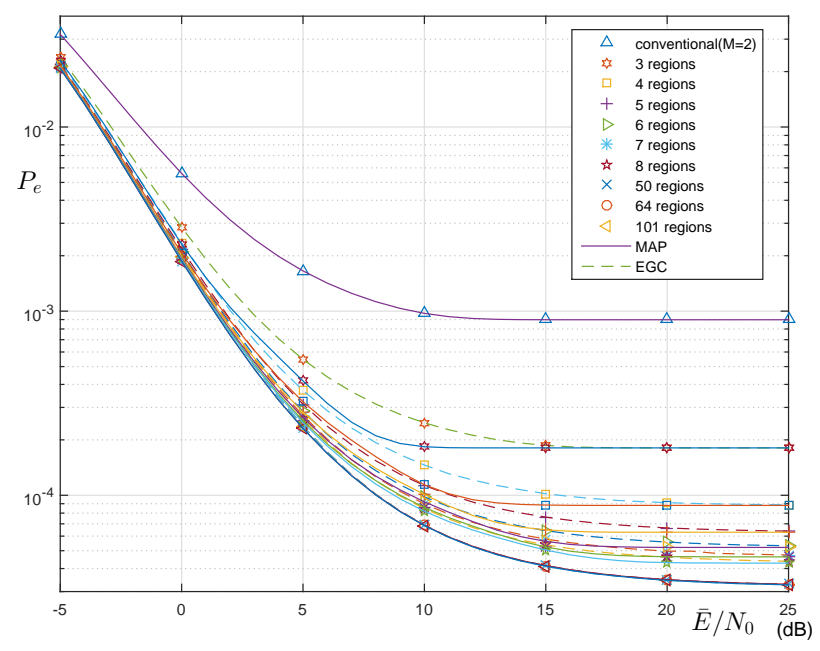

Figure 2. $P_{e}$ of $M S$ with $\gamma=1$ and $L=16$ over $A W G N$.

With $\gamma=1$, the performance of MS under EGC and KB is compared in Figure 3. A crossover exists between MS and KB as SNR increases with KB under either Gray or regular coding. This is because the effect of an erroneous signal sent by the sensor is alleviated in the hard-decision process of the received bit in KS when SNR is high. Regular coding outperforms Gray coding with $k=2,3,6$. This is due to the property of Gray code that the nearest codewords differ in one single bit and, nevertheless, most of the codewords differing in one single bit are not the nearest neighbors, especially when $k$ is large. The corresponding curves of MS and KB emerge since no mistakes occur at the transmission part as SNR increases.

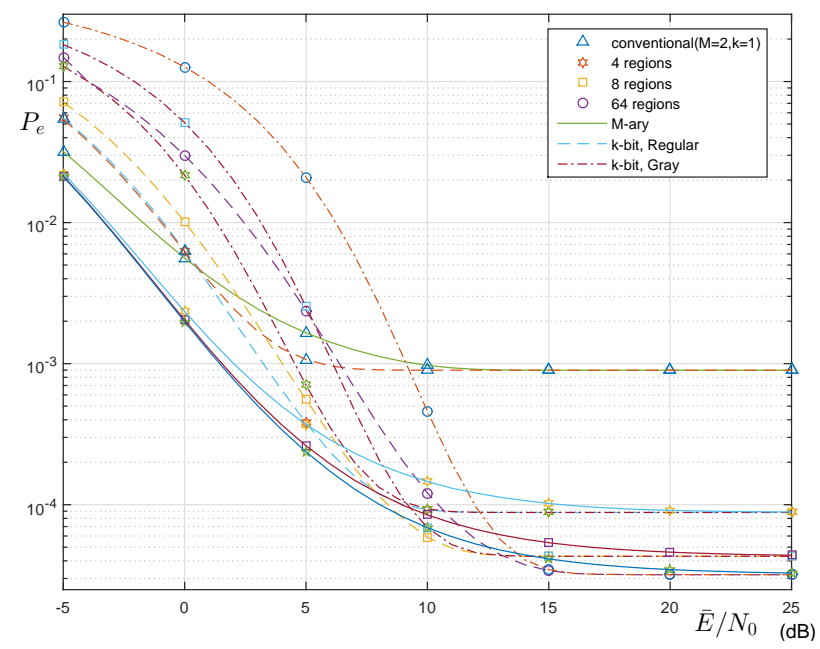

Figure 3. $P_{e}$ of $M S$ and $K B$ with $\gamma=1$ and $L=16$ over $A W G N$.

The comparison of KB decoded under the conditional mean and under the log-likelihood is shown in Figure 4. No significant difference is observed between the two algorithms, which indicates, unlike the MAP rule in MS, sharing the information of $p_{n}$ 's at the FC does not necessarily improve the performance of $\mathrm{KB}$. 


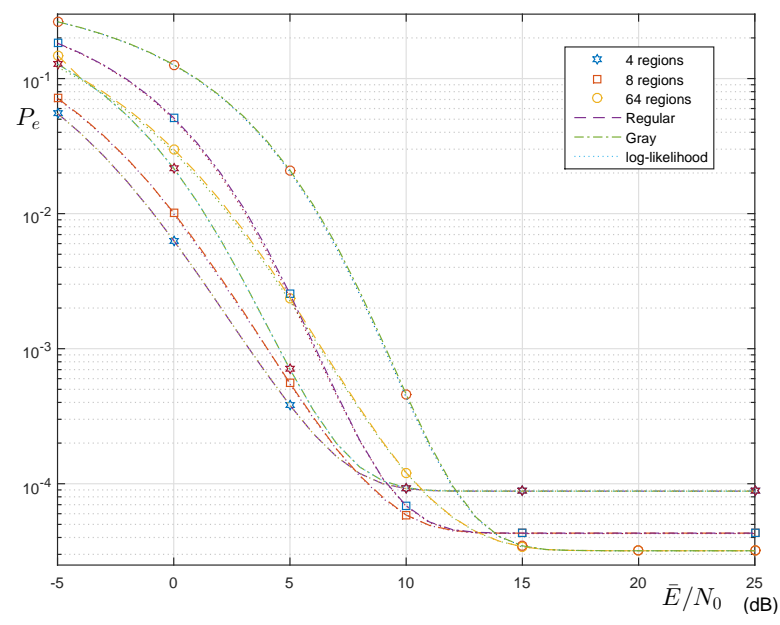

Figure 4. KB under Conditional Mean and under Log-likelihood with $\gamma=1$ and $L=16$ over $A W G N$.

Figure 5 shows the error performance with $\gamma=5$. No obvious difference exists between the behaviors of MS under either the MAP or the EGC rules for $M=2$ to 101 . When $\gamma$ is large, the uncertainty of the decision a sensor makes on its observation is extremely low, and no significant gain exists on

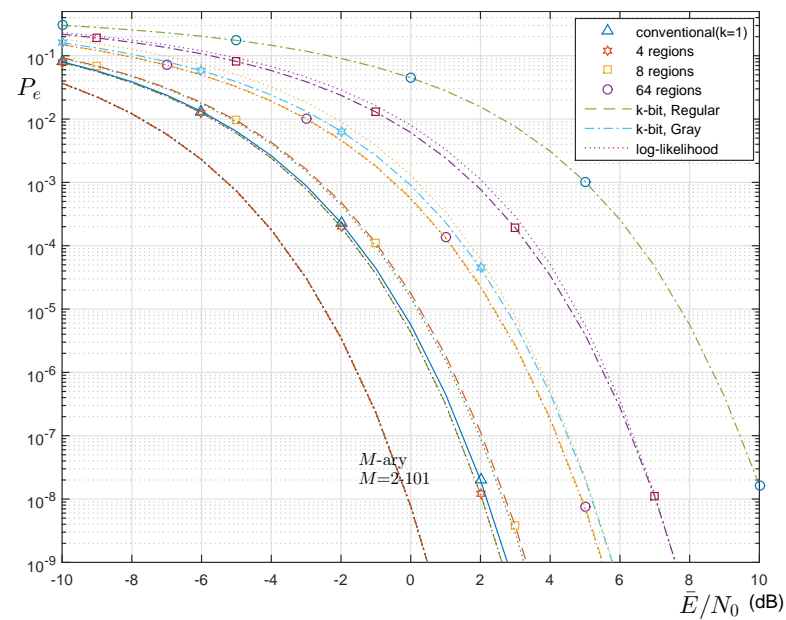

Figure 5. $P_{e}$ of $M S$ and $K B$ with $\gamma=5$ and $L=16$ over $A W G N$.

the performance of MS, whatever value $M$ is. The quantization $X$ by the sensor is always the two end points, i.e., $\mathrm{P}\left[X= \pm x_{N}\right] \simeq 100 \%$. $\Delta$ tends to be tiny and the two end regions $I_{-N}$ and $I_{N}$ dominate the decision on the observation and the MS scheme is virtually binary. Nevertheless, the performance of $\mathrm{KB}$ is worse than that of MS and deteriorates generically as $k$ increases. This is due to the spreading of the energy on the multiple transmitted bits and the innate disadvantage of the hard-decision decoding on the received bits at the FC.

With $\gamma=1$, Figure 6 shows the performance of MS under EGC and KB over a Rayleigh faded channel. $P_{e}$ is improved as $M$ or $k$ Increases. KB outperforms MS at high SNR because when the fading is critical, the reliability of the hard-decision decoding in $\mathrm{KS}$ is higher than that from the combination of the soft information in MS.

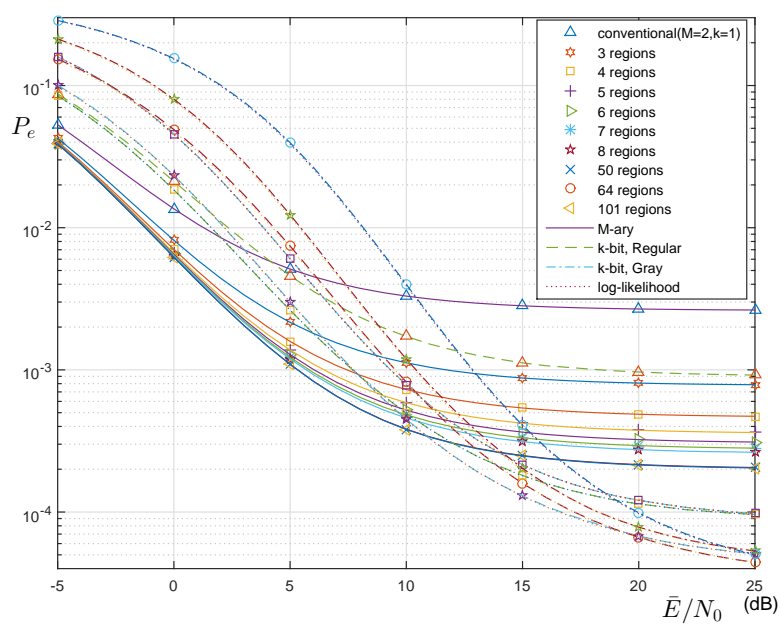

Figure 6. $P_{e}$ of $M S$ and $K B$ with $\gamma=1$ and $L=16$ over Rayleigh Faded Channel.

Finally, the error rates with $\gamma=5$ over a Rayleigh faded channel are shown in Figure 7. Note the similarity between the performances of AWGN channel and of Rayleigh faded channel. When $\gamma$ is large and the uncertainty of a sensor's decision is low, likewise the MS scheme is virtually binary and the performance of $\mathrm{KB}$ is worse than that of MS when the signal is transmitted over a fading channel.

\section{Conclusions}

We have analyzed and compared the performances of MS and $\mathrm{KB}$ under multi-level decisions for distributed binary detection. The decision errors at the FC is minimized via optimizing the region allocation. In addition, the comparison of Gray coding and regular coding in $\mathrm{KB}$ has been made, and the performances of the proposed schemes over an AWGN channel and a fading channel have been both examined. The results confirm that the goal of better error performance under the MS and the KB algorithms is achieved, especially when the certainty of the detection made by the sensor on the environment is low.

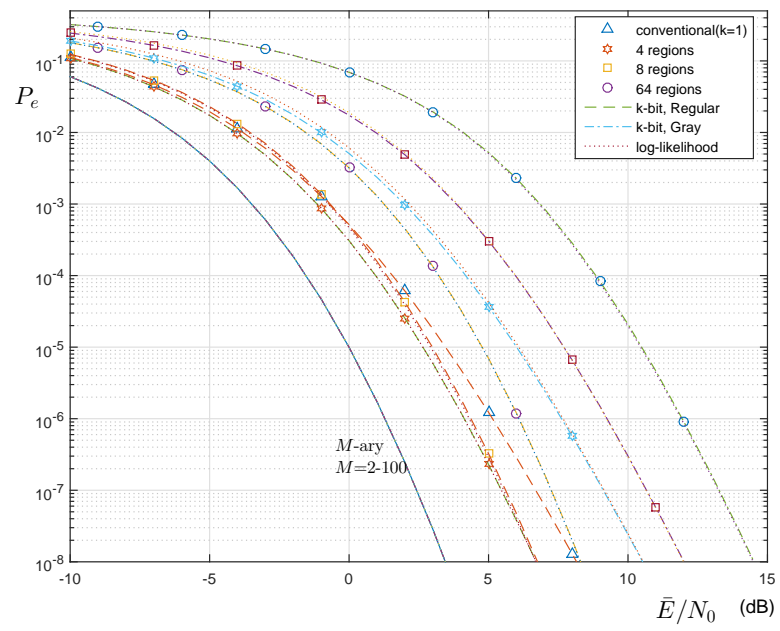

Figure 7. $P_{e}$ of MS and KB with $\gamma=5$ and $L=16$ over Rayleigh Faded Channel. 


\section{References}

[1] S. Chaudhari, J. Lunden, V. Koivunen, and H. V. Poor, "Cooperative sensing with imperfect reporting channels: Hard decisions or soft decisions?" IEEE Transactions on Signal Processing, vol. 60, no. 1, pp. 18-8, January 2012.

[2] S. Saha, A. Kumar, Priyanka, and R. Bhattacharya, "An LLR based cooperative spectrum sensing with hardsoft combining for cognitive radio networks," in 32nd International Union of Radio Science General Assembly Scientific Symposium (URSI GASS), Montreal, Canada, August 2017.

[3] A. K. Kattepur, A. T. Hoang, Y.-C. Liang, and M. J. Er, "Data and decision fusion for distributed spectrum sensing in cognitive radio networks," in 2007 6th International Conference on Information, Communications Signal Processing, Singapore, December 2007.

[4] Q. Cheng, P. K. Varshney, J. H. Michels, and C. M. Belcastro, "Fault detection in dynamic systems via decision fusion," IEEE Transactions on Aerospace and Electronic Systems, vol. 44, no. 1, pp. 227-242, January 2008.

[5] Q. Ling, Z. Tian, and Y. Li, "Distributed decision-making in wireless sensor networks for online structural health monitoring," Journal of Communications and Networks, vol. 11, no. 4, pp. 350-358, August 2009.

[6] H. Wang, J. Elson, L. Girod, D. Estrin, and K. Yao, "Target classification and localization in habitat monitoring," in IEEE International Conference on Acoustics, Speech, and Signal Processing (ICASSP 2003), Hong Kong, China, April 2003.

[7] L. Dan, K. D. Wong, H. H. Yu, and A. M. Sayeed, "Detection, classification, and tracking of targets," IEEE Signal Processing Magazine, vol. 19, pp. 17-29, March 2002.

[8] E. Nurellari, D. McLernon, and M. Ghogho, "Distributed two-step quantized fusion rules via consensus algorithm for distributed detection in wireless sensor networks," IEEE Transactions on Signal and Information Processing over Networks, vol. 2, no. 3, pp. 321-335, September 2016.

[9] R. S. Blum, "Distributed detection for diversity reception of fading signals in noise," IEEE Transactions on Information Theory, vol. 45, no. 1, pp. 158-164, January 1999.

[10] P. K. Varshney, Distributed Detection and Data Fusion. New York: Springer, 1997.
[11] S. C. A. Thomopoulos, R. Viswanathan, and D. C. Bougoulias, "Optimal decision fusion in multiple sensor systems," IEEE Transactions on Aerospace and Electronic Systems, vol. AES-23, no. 5, pp. 644-653, September 1987.

[12] M. Xiang and C. Han, "Global optimization for distributed detection system under the constraint of likelihood ratio quantizers," in Proceedings of the Third International Conference on Information Fusion, Paris, France, July 2000.

[13] M. Xiang and C. Han, "Global optimization for distributed and quantized bayesian detection system," in Proceedings of the Third International Conference on Information Fusion, Paris, France, July 2000.

[14] H. Varaee, G. Mirjalily, and A. Pouramini, “An analytical technique to determine the decision thresholds of multibit distributed detection in sensor networks," in 2009 Second International Conference on Computer and Electrical Engineering, Dubai, United Arab Emirates, December 2009.

[15] X. Fan, D. Duan, and L. Yang, "Multi-bit cooperative spectrum sensing strategy in closed form," in 2013 Asilomar Conference on Signals, Systems and Computers, Pacific Grove, CA, November 2013.

[16] A. M. Aziz, "A simple and efficient suboptimal multilevel quantization approach in geographically distributed sensor systems," Signal Processing, vol. 88, no. 7, pp. 1698-1714, July 2008.

[17] A. M. Aziz, "A soft-decision fusion approach for multiple-sensor distributed binary detection systems," IEEE Transactions on Aerospace and Electronic Systems, vol. 47, no. 3, pp. 2208-2216, July 2011.

[18] R. Viswanathan and P. K. Varshney, "Distributed detection with multiple sensors part i. fundamentals," Proceedings of the IEEE, vol. 85, no. 1, pp. 54-63, January 1997.

[19] Q. Zhang and P. K. Varshney, "Decentralized Mary detection via hierarchical binary decision fusion," Information Fusion, vol. 2, pp. 3-16, 2001.

[20] M. Longo, T. D. Lookabaugh, and R. M. Gray, "Quantization for decentralized hypothesis testing under communication constraints," IEEE Transactions on Information Theory, vol. 36, no. 2, pp. 241-255, March 1990.

[21] R. Krzysztofowicz and D. Long, "Fusion of detection probabilities and comparison of multisensor systems," IEEE Transactions on Systems, Man, and Cybernetics, vol. 20, no. 3, pp. 665-677, May/June 1990. 
[22] C. W. Helstrom, "Gradient algorithm for quantization levels in distributed detection systems," IEEE Transactions on Aerospace and Electronic Systems, vol. 31, no. 1, pp. 390-398, January 1995.

[23] V. A. Aalo and R. Viswanathan, "Multilevel quantisation and fusion scheme for the decentralised detection of an unknown signal," IEE Proceedings - Radar, Sonar and Navigation, vol. 141, no. 1, pp. 37-44, February 1994.

[24] V. Cheng and T. Wang, "Performance Analysis of Distributed Decision Fusion Using A Censoring Scheme in Wireless Sensor Networks," IEEE Trans. Vehicular
Technology, vol. 59, no. 6, pp.2845-2851, July 2010.

[25] V. Cheng and T. Wang, "Performance Analysis of Distributed Decision Fusion Using A Multilevel Censoring Scheme in Wireless Sensor Networks," IEEE Trans. Vehicular Technology, vol. 61, no. 4, pp.16101619, May 2012.

[26] V. Cheng, T. Wang, and H. Wang, "Distributed Decision Fusion With M-ary Source Coding On Sensor Observation and Uncoded Data Transmission," in IEEE Consumer Communications \& Networking Conference(CCNC), Las Vegas, USA, January 2020. 